\title{
DIAGNÓSTICO, ESTADIAMENTO E TRATAMENTO CIRÚRGICO DO ADENOCARCINOMA DE PÂNCREAS
}

\author{
Diagnosis, staging and treatment of pancreatic adenocarcinoma
}

\author{
Enio Campos AMICO, Élio Jose Silveira da Silva BARRETO, \\ Antônio Medeiros DANTAS-FILHO, Irami ARAÚJO-FILHO
}

ABCDDV/623

Amico EC, Barreto JSS, Dantas-Filho AM, Araújo-Filho I. Diagnóstico, estadiamento e tratamento cirúrgico do adenocarcinoma de pâncreas. ABCD Arq Bras Cir Dig 2008;21(4): 192-200

RESUMO - Introdução - O adenocarcinoma pancreático é um dos tumores sólidos de pior prognóstico, sendo o tratamento cirúrgico o único potencialmente curativo. Na grande maioria dos pacientes o tumor é diagnosticado em fase avançada, comumente na presença de doença metastática. A introdução de modernos métodos diagnósticos associados ao aperfeiçoamento dos já existentes tem gerado controvérsia quanto à melhor maneira de se estabelecer o diagnóstico e estadiamento do tumor. Da mesma forma, o papel da cirurgia na paliação e aspectos técnicos da ressecção de lesões localizadas estão longe de alcançarem consenso na prática. Método - Revisão da literatura sobre os aspectos controversos relacionados ao tema e um algoritmo para a abordagem dos pacientes com suspeita de tumor de pâncreas são apresentados. Foram utilizados os descritores: "adenocarcinoma" e "pâncreas" para pesquisa no PubMed (www.pubmed.com) e na Bireme (www.bireme.br) e a seguir selecionadas as publicações pertinentes a cada tópico escolhido com atenção especial para metanálises, estudos clínicos controlados, revisões sitemáticas e ainda publicações de grandes centros especializados em doenças pancreáticas. Conclusões - Na suspeita de adenocarcinoma de pâncreas é possível realizar estadiamento muito próximo do real sem a necessidade da exploração cirúrgica sistemática em virtude da disponibilidade na prática de exames modernos e eficientes. Isso permite que paliação menos invasiva seja praticada na maioria dos pacientes com lesões avançadas e incuráveis. Nos em que a cura é possível, a operação deve ser realizada objetivando-se, essencialmente, a remoção da lesão com margens livres e com aceitáveis índices de morbi-mortalidade.

DESCRITORES - Pâncreas. Adenocarcinoma. Diagnóstico. Cirurgia.

\section{INTRODUÇÃO}

O adenocarcinoma de pâncreas (AP) persiste como um dos mais agressivos tumores malignos do trato digestivo. Isso pode ser facilmente demonstrado pela estreita correlação entre o número de novos casos diagnosticados e a incidência de morte pela doença por ano nas várias regiões do mundoํ. Felizmente no Brasil essa doença não figura entre as mais prevalentes causas de câncer, sendo responsável apenas por $4 \%$ das mortes por neoplasia maligna em nosso país ${ }^{15}$. A despeito disso os esforços se concentram para que a cura, possível em minoria dos pacientes, e a melhoria da qualidade de vida, naqueles com doença avançada localmente ou metastática, seja oferecida aos pacientes nos quais o diagnóstico de AP é feito.

Admite-se que a única alternativa para a sua cura seja o tratamento cirúrgico. Ainda assim, apenas poucos

Trabalho realizado no Departamento de Medicina Clínica e Departamento de Cirurgia do Hospital Universitário Onofre Lopes - Universidade Federal do Rio Grande do Norte, Natal, RN, Brasil.

Correspondência: Enio CamposAmico,e-mail: ecamic@uol.com.br pacientes submetidos à pancreatectomia sobrevivem mais que cinco anos. Mesmo naqueles pacientes com tumores pequenos, submetidos à ressecção com margens negativas e com gânglios livres de tumor a taxa de sobrevida em cinco anos alcança apenas $41 \%{ }^{5}$. Para a maioria dos pacientes, no entanto, a cura não pode ser oferecida. Quando analisado a totalidade dos pacientes, apenas em $15-20 \%$ dos casos a ressecção é possível em virtude de doença avançada localmente ou pela presença de metástase à distância. Nesses casos é esperada sobrevida média de aproximadamente seis meses ${ }^{33}$.

Em virtude dessa realidade, o cirurgião tem papel central na abordagem do AP sendo seus objetivos: 1) promover a ressecção cirúrgica com margens livres e 2) proporcionar um mínimo possível de morbidade e mortalidade com o tratamento. Acrescenta-se a isso a participação no processo decisório quanto à melhor paliação possível a ser aplicada aos pacientes que pode ser realizada de forma cirúrgica ou endoscópica.

Frente à possibilidade do diagnóstico e tratamento alguns aspectos persistem controversos enquanto outros estão mais bem definidos. Esta revisão aborda o estado 
atual da abordagem do AP e ao final é apresentada proposta de algoritmo para a sua abordagem.

\section{MÉTODO}

Foram utilizados os descritores: "adenocarcinoma" e "pâncreas" para pesquisa no PubMed (www.pubmed.com) e na Bireme (www.bireme.br) e a seguir selecionadas as publicações pertinentes a cada tópico escolhido com atenção especial para metanálises, estudos clínicos controlados, revisões sitemáticas e ainda publicações de grandes centros especializados em doenças pancreáticas.

\section{REVISÃO DA LITERATURA}

\section{Diagnóstico}

\section{Massa pancreática: biópsia ou ressecção?}

A apresentação clínica mais comum nos pacientes portadores de AP é o aparecimento de icterícia no sexo masculino a partir da sexta década de vida no qual uma massa em cabeça pancreática é encontrada ao exame de imagem. Tradicionalmente a partir daí o diagnóstico é suspeitado e a pancreatectomia é programada. O principal argumento que suporta essa conduta é a alta incidência do adenocarcinoma nesses pacientes. Smith et al. ${ }^{41}$ avaliando retrospectivamente 603 pacientes na Clínica Mayo, submetidos à duodenopancreatectomia com massas pancreáticas suspeitas de AP, em período de 34 anos na instituição, encontraram em apenas 29 pacientes (5\%) outros diagnósticos sendo a pancreatite crônica o mais freqüente ${ }^{41}$.

Existem algumas possibilidades para obtenção de material citológico ou histológico para o diagnóstico. São eles: punção guiada por ecoendoscopia, punção guiada por tomografia de abdome, punção obtida por laparoscopia e escovado obtido pelo exame de endoscopia.

Com o advento da ecoendoscopia e com ela a possibilidade de punção das massas pancreáticas a obrigatoriedade de obtenção de material para confirmação histo-patológica do AP têm sido sugerida por alguns autores. A principal vantagem com essa abordagem é de se evitar o tratamento cirúrgico em um pequeno grupo de pacientes que de outra forma seriam submetidos aos altos riscos da pancreatectomia. Para análise da questão é necessário que se conheçam: 1) qual a acurácia da punção nos pacientes portadores de massas pancreáticas? e 2) qual o risco de morte nos pacientes submetidos à pancreatectomia?

A sensibilidade da punção com agulha fina guiada por ecoendoscopia (PAF-EE) varia de 75-80\% com especificidade próxima de $100 \%$. Alguns fatores como experiência do médico e a presença do cito-patologista na sala de endoscopia tornam o método ainda mais eficiente ${ }^{36}$. Em recente consenso brasileiro de ecoendoscopia, com nível de evidência 1, a sensibilidade do método para a detecção de nódulos pancreáticos mesmo nas lesões menores de 2 $\mathrm{cm}$, foi acima de $90 \%{ }^{22}$. O problema crucial que se impõem quando se inclui a PAF-EE no algoritmo da abordagem da massa pancreática é a possibilidade do resultado falso negativo. Di stasi et al. ${ }^{10} \mathrm{em}$ estudo multicêntrico coletando 323 pacientes com diagnóstico de $\mathrm{AP}$ submetidos à $\mathrm{PAF}-\mathrm{EE}$ encontraram resultado falso negativo para câncer ou inconclusivo para diagnóstico em $14 \%$ dos $\operatorname{casos}^{10}$. Consenso publicado em 2005 envolvendo cinco instituições britânicas de gastroenterologia sugere que na impossibilidade de se fazer diagnóstico histopatológico de lesões pancreáticas suspeitas, o tratamento cirúrgico não deve ser retardado o que anula o valor do método em evitar o procedimento cirúrgico ${ }^{29}$.

Muito tem sido publicado principalmente na literatura médica norte-americana quanto à regionalização nos procedimentos de pancreatectomia. Isso tem sido justificado em virtude de resultados superiores quando esse procedimento é realizado em hospital com grande volume de procedimentos por ano. Desta forma, nesses hospitais, taxa de mortalidade entre 1 e $4 \%^{2}$ tem sido obtida o que pode ser considerado grande avanço quando se compara com mortalidade em torno de $25 \%$ nos anos 60 .

Modelo muito interessante de análise da questão sobre a política de biópsia mandatória pré-operatória foi apresentado por Wolfson et a $l^{50}$. A comparação hipotética entre 10.000 pacientes submetidos à biópsia pré-operatória e operados no caso de positividade desta e outros 10.000 pacientes submetidos a tratamento cirúrgico por portarem massas pancreáticas nos quais a biópsia foi dispensada, aponta para um número de vidas salvas quatro vezes maior no grupo que dispensou a realização da biópsia. Esse dado foi obtido utilizando taxa de $10 \%$ de falso negativo para as biópsias guiadas por ecoendoscopia e índice de mortalidade de $2 \%$ para as ressecções pancreáticas ${ }^{50}$.

Baseado nos dados apresentados e apesar dos altos índices de sensibilidade da PAF-EE ainda se justifica a realização da pancreatectomia na massa pancreática independente da realização ou do resultado da biópsia pré-operatória. Isso é particularmente verdadeiro quando se pode oferecer aos pacientes baixo índice de mortalidade com o tratamento cirúrgico. Para os pacientes com massas pancreáticas assintomáticas, no entanto, com risco cirúrgico aumentado, a realização da PAF-EE pode ser útil em modificar a programação cirúrgica por vezes até evitando a operação ${ }^{50}$.

\section{O papel da laparoscopia}

Ela no estadiamento do AP permanece controverso. Para os pacientes considerados ressecáveis pelos exames de imagem não-invasivos, questiona-se se a laparoscopia deva ser feita para todos os pacientes, seletivamente ou para nenhum paciente. A despeito do avanço tecnológico dos exames pré-operatórios, vários autores mostram alta incidência de casos com estadiamento intra-operatório modificado em virtude de doença locorregional avançada ou pelo diagnóstico de metástases à distância. Estima-se que a laparoscopia modifique o estadiamento dos pacientes portadores de AP, evitando operação desnecessária em pelo menos $20 \%$ dos pacientes ${ }^{7}$. 
A principal vantagem com a laparoscopia parece ser obtida naqueles casos de pequenos implantes $(<1 \mathrm{~cm})$ na superfície hepática ou em peritônio que normalmente não são encontrados pelos exames de imagem pré-operatórios e que facilmente são identificados e biopsiados pela via laparoscópica. A adição do ultrassom laparoscópico permite ainda o estudo das relações do tumor com o eixo mesentérico-portal e artéria mesentérica superior, permitindo ganho na comparação com a laparoscopia convencional ${ }^{23}$.

O principal argumento contrário à realização da laparoscopia no estadiamento do AP é a possibilidade de se promover a paliação cirúrgica através da derivação biliar e gástrica durante o mesmo ato operatório em que se avalia a ressecabilidade da lesão. Com a possibilidade de utilização de próteses pela via endoscópica, no entanto, a necessidade de derivação cirúrgica tornou-se menos imperiosa que no passado. A derivação cirúrgica pelo acesso laparoscópico durante o procedimento de estadiamento também, hoje, é uma realidade e opção aceita para a paliação do $\mathrm{AP}^{43}$.

Recentes estudos têm tentado identificar variáveis pré-operatórias que possam apontar para um grupo de pacientes com alta probabilidade de metástases. Desta forma, pacientes com tumores maiores de $3 \mathrm{~cm}$, em topografia de corpo-cauda e ainda aqueles com Ca 19-9 teriam maior probabilidade de serem beneficiados ${ }^{26,37}$. Uma vez que se consiga selecionar um grupo específico de pacientes com alta probabilidade de portarem doença avançada, o que elevaria os índices de eficácia da laparoscopia em evitar procedimento cirúrgico, é provável que a laparoscopia seja aceita como procedimento que preceda a exploração cirúrgica aberta.

\section{Citologia}

É controverso se o diagnóstico de citologia positiva no lavado intra-perironeal em pacientes portadores de AP indica doença metastática e portanto irressecável.

Yachida et al..$^{51}$. estudando 134 pacientes submetidos à ressecção pancreática, encontraram citologia positiva em $14 \%$. Embora nesses pacientes tumores maiores e mais avançados tenham sido descritos quando comparados com aqueles nos quais a citologia foi negativa, a sobrevida global entre os grupos não alcançou diferença estatística ${ }^{51}$. Meszoely et al. ${ }^{24}$ de forma semelhante com casuística muito similar (135 pacientes ressecados) também não encontrou diferença na detecção de recorrência e na sobrevida entre os grupos com citologia positiva ou negativa sob as mesmas condições ${ }^{24}$.

Em estudo mais recente realizado por Ferrone et al. ${ }^{13}$, foram analisados 462 pacientes portadores de AP e submetidos a citologia intra-peritoneal. Analisando globalmente os pacientes com positividade no exame citológico, foi observado em $17 \%$ correlação com o estágio da doença. Enquanto naqueles pacientes com doença localmente avançada ou metastática a sobrevida foi indiferente quando a positividade no exame citológico, nos pacientes com tumores considerados ressecáveis houve diferença estatística a favor do grupo com citologia negativa. Os autores sug- erem a partir desses dados que a laparoscopia com citologia intra-peritoneal deva ser realizada como procedimento separado em pacientes de alto risco ${ }^{13}$.

Em virtude da descrição de pacientes submetidos à ressecção e que tiveram sobrevida prolongada na vigência de citologia positiva e ainda em virtude da necessidade de procedimento cirúrgico adicional para coleta de liquido para exame citológico, parece prudente aguardar estudos definitivos antes de se contraindicar a ressecção em pacientes com doença ressecável e citologia positiva para tumor.

\section{Estadiamento}

\section{Exames pré-operatórios}

A finalidade dos exames de imagem aplicados ao AP são: 1) fornecer o diagnóstico, 2) avaliar a invasão locorregional da lesão (ressecabilidade) e 3 ) avaliar a presença de doença à distância. A tomografia de abdome com múltiplos detectores, a ressonância nuclear magnética a ecoendoscopia, a tomografia com emissão de pósitrons e a laparoscopia preenchem em maior ou menor grau esses objetivos.

Em virtude das várias possibilidades entre os exames diagnósticos, é importante que seja levado em conta a sequência mais efetiva com menor custo. Infelizmente existem poucas publicações comparativas com essa finalidade na abordagem do AP uma vez que a maioria das publicações são séries de casos submetidos preferencialmente a um tipo específico de método da preferência dos autores.

De acordo com várias publicações, a tomografia de abdome com múltiplos detectores é na atualidade o exame preferido para o diagnóstico e estadiamento do $\mathrm{AP}^{2,6,30,34,35}$. Ela permite exame rápido em um único evento de apnéia, com fases arterial, portal e parenquimatosa, com cortes finos e reconstrução em vários planos. Desta forma, é obtida precisa correlação do tumor com estruturas próximas como o tronco mesentérico-portal, artéria mesentérica superior e ainda órgãos distantes, como o fígado podem ser adequadamente avaliado.

Em um dos poucos estudos comparativos, Soriano et al. ${ }^{42}$ estudaram 62 pacientes submetidos à cirurgia que previamente ao procedimento cirúrgico foram submetidos à tomografia computadorizada helicoidal, ressonância nuclear magnética, arteriografia e ecoendoscopia. A tomografia computadorizada helicoidal demonstrou a mais alta acurácia quanto à extensão do tumor primário (73\%), extensão locorregional (74\%), invasão vascular $(83 \%)$, metástase à distância (83\%), estadiamento TNM (46\%) e ressecabilidade (83\%) enquanto a ecoendoscopia foi superior na avaliação do tamanho do tumor e comprometimento linfonodal ${ }^{42}$.

Descrita em 1980 e popularizada nos anos subsequentes, a ecoendoscopia tem papel importante na investigação do AP. As principais indicações correntes para os pacientes com AP são: detecção de massa pancreática e necessidade de biópsia de tecido pancreático. Em estudo prospectivo comparativo entre a tomografia de abdome com múltiplos 
detectores e a ecoendoscopia, DeWitt et al. ${ }^{9}$, analisando 80 pacientes portadores de $\mathrm{AP}$, observaram superior sensibilidade na detecção do tumor com a ecoendoscopia. A ressecabilidade da lesão foi identificada de com a mesma eficiência entre os dois métodos (88\% - ecoendoscopia e $92 \%$ - tomografia de abdome com múltiplos detectores). $\mathrm{Na}$ análise dos pequenos tumores, aqueles menores de $25 \mathrm{~mm}$, não houve diferença estatística na comparação com os métodos o que pode ter sido consequência do pequeno número de pacientes incluídos nessas situação. A despeito disso os autores sugerem a utilidade da ecoendoscopia naqueles pacientes nos quais a tomografia de abdome com múltiplos detectores não identifica a lesão9 ${ }^{9}$ É importante considerar, no entanto, o maior custo e menor disponibilidade em nosso meio da ecoendoscopia à favor da tomografia de abdome com múltiplos detectores. Ela é exame não-invasivo que a partir do uso de um análogo da glicose marcado com radioisótopo permite a análise do metabolismo de células normais e tumoras, diferenciando-as. Permite a varredura do corpo inteiro e é utilizado muito frequentemente na análise de tumores como o adenocarcinoma de cólon. Recentemente, desde 2000, com a finalidade de tornar o exame mais preciso do ponto de vista anatômico, mais rápido e possibilitar a mensuração das imagens, a tomografia foi associada ao tomografia com emissão de pósitrons.

O principal uso dela estadiamento do AP é para aqueles casos nos quais o sugere-se lesão ressecável. Em uma parte desses pacientes, a operação poderia ser evitada pelo diagnóstico de metástase à distância. Heinrich et al. ${ }^{14}$ estudaram 59 pacientes com suspeita de AP que foram submetidos à tomografia de abdome com múltiplos detectores, radiografia de tórax, ecoendoscopia, Ca 19-9, laparoscopia e tomografia com emissão de pósitrons. Esta diagnosticou metástase à distância em 13 de 16 pacientes sendo que em cinco todos os demais exames mostravam-se normais. Em consequência disso, $16 \%$ dos pacientes tiveram a conduta modificada pela adição deste exame. Embora a partir da análise de custos intra-hospitalares, os autores tenham considerado o exame custo-efetivo, na maioria dos centros, em virtude do seu pequeno impacto na população total de pacientes portadores de AP a realização do tomografia com emissão de pósitrons ainda não foi incorporado ao estadiamento do $\mathrm{AP}^{14}$.

\section{Tratamento cirúrgico}

\section{Paliação: prótese endoscópica ou derivação cirúrgica?}

O tratamento cirúrgico paliativo para os portadores de AP com doença irressecável ou metastática tradicionalmente inclui a anastomose bilio-digestiva (na presença de icterícia), a gastro-enteroanastomose (para tratamento ou prevenção da obstrução duodenal) e ainda a alcoolização do plexo celíaco - esta última em alguns casos. O uso efetivo das próteses biliares endoscópicas na obstrução maligna, associada ao fato de serem desnecessários a maioria dos procedimentos de derivação gástrica realizados cirurgicamente tornaram defensável a paliação do AP de forma não operatória.

Em um estudo que avaliou 155 pacientes nos quais o procedimento de laparoscopia para estadiamento do AP revelou doença irressecável ou metastática, Espat e cols. observaram que em apenas $2 \%$ dos pacientes houve a necessidade de procedimento cirúrgico paliativo. Nesse estudo, 56 pacientes foram submetidos à derivação biliar por próteses em virtude da presença de icterícia, 21 dos quais após o procedimento laparoscópico. Embora não tenha sido especificado o local do tumor (cabeça ou corpo/ cauda) a presença de icterícia em apenas $32 \%$ dos pacientes já portadores de doença avançada no momento do diagnóstico sugere maior frequência de tumores em topografia de corpo/cauda o que pode justificar baixa incidência de sinais de obstrução duodenal ${ }^{12}$.

Em estudo prospectivo e randomizado com 30 pacientes portadores de AP metastático, submetidos à paliação endoscópica ou cirúrgica, Artifon et al. ${ }^{3}$ observaram menor custo geral (incluindo o procedimento, internação hospitalar, cuidados pós-drenagem e tratamento de complicações) associado à melhor qualidade de vida através de questionário aplicado com 30, 60 e 120 dias, nos pacientes submetidos à derivação endoscópica com prótese metálica. Embora não tenha havido diferença estatística quanto à incidência de complicações com os dois procedimentos, $o$ estudo aponta para maior número de complicações severas com a operação. Também foi relevante nessa casuística que nenhum dos pacientes do grupo endoscópico tenha apresentado obstrução duodena ${ }^{13}$.

As próteses auto-expansivas de metal têm sido preferidas no manuseio dos pacientes com obstrução maligna da via biliar em virtude do seu maior diâmetro e consequente mais eficaz drenagem com menor necessidade de revisões para desobstrução. Esse material, no entanto, apresenta custo muito superior que àquelas de plástico. Desta forma estratégia defendida por Kaassis et al. ${ }^{16}$ contempla a utilização de próteses metálicas na ausência de metástases hepáticas onde se espera sobrevida de superior à três meses ${ }^{16}$.

\section{Paliação: dupla derivação cirúrgica?}

Uma vez que a exploração cirúrgica tenha sido indicada com intenção curativa no AP de topografia cefálica, é possível que o estadiamento intra-operatório evidencie doença avançada que de alguma maneira contraindique a ressecção. Nessa situação o procedimento paliativo recomendado deve incluir a derivação biliar na presença de icterícia obstrutiva, e gástrica para prevenção da obstrução duodenal. Essa recomendação é baseada em dois estudos prospectivos e randomizados que concluíram ser a dupla derivação eficaz em prevenir sintomas de obstrução gástrica, com morbidade similar ${ }^{20,47}$.

É provável que os pacientes que tem a ressecção cirúrgica contraindicada durante a exploração cirúrgica tenham ainda assim doença menos avançada que aqueles nos quais os exames pré-operatórios já sugerem irressecabilidade ou evidência de metástases à distância. Para esse último grupo menor sobrevida é esperada. Essa talvez seja a explicação 
de menores índices de sintomas de obstrução gástrica nos pacientes que sequer são submetidos à exploração cirúrgica.

\section{Drenagem biliar pré-operatória}

Em virtude da cabeça pancreática ser o local preferencial de acometimento do AP, é muito frequente que os pacientes já apresentem icterícia como sintoma inicial da doença. A drenagem biliar pré-operatória em potencialmente ressecáveis objetiva o alívio do acúmulo de bilirrubina e ácidos biliares, prevenção da colangite, redução da translocação bacteriana, melhora do estado nutricional, alívio do prurido, entre outras vantagens teóricas. $\mathrm{Na}$ prática, no entanto, vários estudos têm atribuído a esse procedimento maior incidência de complicações infecciosas no pós-operatório de ressecções pancreáticas nos portadores de icterícia obstrutiva. Várias publicações existem sobre o tema com resultados contraditórios.

Em metanálise publicada por Sewnath et al.38, onde foram incluídos 23 estudos, e 3155 pacientes, os resultados sugerem que a drenagem pré-operatória não trás benefícios e por isso não deve ser realizada de rotina no préoperatório de pacientes portadores de icterícia obstrutiva. Várias objeções metodológicas impossibilitam adequada conclusão desta metanálise: estudos não randomizados foram incluídos, o AP representou pouco menos da metade dos pacientes arrolados no estudo, o tipo de procedimento cirúrgico não foi uniformizado (pancreatectomias, hepatectomias foram incluídos), um grande número de pacientes foi submetido à drenagem biliar externa com seus riscos superiores à drenagem interna por endoscopia ${ }^{38}$.

Um estudo controlado multi-institucional com inclusão prevista de 210 pacientes nos quais pacientes portadores de tumores peri-ampular serão randomizados a receber drenagem pré-operatória endoscópica (preferencialmente) ou operação imediata está em andamento e espera-se poder esclarecer o controverso papel do alívio da obstrução biliar nos portadores de $\mathrm{AP}^{44}$.

\section{Duodenopancreatectomia com ou sem preservação do piloro?}

A duodenopancreatectomia original, conhecida como procedimento de Kausch-Whipple implica na ressecção do antro gástrico. A outra possibilidade técnica é conhecida como duodenopancreatectomia com preservação do piloro sendo nesta variante técnica a primeira porção duodenal justa-pilórica utilizada para a reconstrução do trânsito. As vantagens da duodenopancreatectomia incluem melhor margem oncológica uma vez que todo o duodeno é removido na peça cirúrgica e ainda menor incidência de retardo de esvaziamento gástrico pós-operatório. Já os defensores da duodenopancreatectomia com preservação do piloro sugerem ser o procedimento mais fisiológico e desta forma teriam os pacientes, menor incidência de síndromes pós-gastrectomia com melhores resultados nutricionais.

$\mathrm{O}$ estudo controlado com o maior número de pacientes comparando a operação de Whipple padrão e a duodeno- pancreatectomia com preservação do piloro foi realizado por Tran et al. ${ }^{45}$. Em estudo multicêntrico incluindo 170 pacientes com tumores de pâncreas e peri-ampulares nenhuma diferença foi encontrada quanto ao tempo operatório, perda de sangue, tempo de internação, morbidade, mortalidade, incidência de retardo de esvaziamento gástrico e sobrevida com os dois tipos de procedimento ${ }^{45}$.

Quando a duodenopancreatectomia com preservação do piloro é preferida é importante que se considere o exame dos gânglios perigástricos na busca de suspeita de metástase linfonodal que pode estar presente em $5 \%$ dos pacientes e ainda é importante o exame de congelação da margem duodenal no intra-operatório ${ }^{52}$.

\section{O papel da linfadenectomia}

O racional para a linfadenectomia na ressecção do AP é o mesmo para a maioria dos tumores sólidos. Objetiva-se com a ressecção de gânglios a remoção de doença locoregional que de outra forma seria deixada no leito do órgão removido. Para que esse raciocínio esteja certo é imperativo que as estações linfonodais funcionem como um bloqueio para a disseminação sistêmica da doença. Baseado em alguns estudos asiáticos retrospectivos na década de 80 , a eficácia da linfadenectomia no AP foi estabelecida e praticada em vários centros deste então. A partir do final da década de 90, no entanto, os primeiros trabalhos prospectivos e randomizados foram publicados.

Pedrazzoli et al. ${ }^{31}$ randomizaram 81 pacientes que à ressecção com linfadenectomia padrão ou extendida que incluía remoção de gânglios ao redor da aorta, artéria mesentérica superior e tronco celíaco. Não houve diferença quanto à morbidade, mortalidade ou sobrevida entre os grupos. Os autores encontraram melhor sobrevida, no entanto, no subgrupo de pacientes submetidos à linfadenectomia alargada com gânglios positivos para tumor ${ }^{31}$.

Na maior casuística já publicada sobre o tema, Yeo et al. ${ }^{35}$ estudaram 294 pacientes portadores de tumores peri-ampulares submetidos à linfadenectomia padrão e extendida. Das variáveis estudadas apenas foi observado diferença entre os grupos quanto à morbidade que foi superior no grupo da linfadenectomia extendida (29\% x $43 \%$ ) como consequência de maiores índices de retardo do esvaziamento gástrico e de fístula pancreática nesse grupo de pacientes ${ }^{35}$.

Em estudo multicêntrico japonês realizado por Nimura et al. ${ }^{28}$, à semelhança do estudo anterior, foi encontrado maior morbidade no grupo de pacientes submetidos à linfadenectomia ampliada em decorrência principalmente de alta incidência de diarréia nesse grupo de pacientes. Mais uma vez nenhuma vantagem quanto à sobrevida foi encontrada ${ }^{28}$.

Em recente publicação, Michalki et al..$^{25}$, apresentaram revisão sistemática e metanálise que envolveu 484 potenciais estudos sobre a linfadenectomia para o AP. Em suas conclusões, sugerem que a linfadenectomia, se muito, deve ser restrita apenas a estudos controlados ${ }^{25}$. 


\section{Uso de octreotide?}

Em virtude do poder corrosivo do suco pancreático em contato com os órgãos abdominais, a fístula do coto pancreático é uma das maiores causas de complicações e de morte após a cirurgia pancreática por esse motivo a redução da incidência da fístula pancreática é altamente desejada. A somatostatina e seus análogos, têm sido usados no intra e pós-operatório das pancreatectomias em virtude de seus efeitos na redução da secreção pancreática exócrina. Múltiplos estudos controlados randomizados existem disponíveis na literatura médica para a análise da eficácia dessas drogas. Estudos clínicos inicias utilizando o octreotide foram realizados na Europa e seus resultados apontaram para a redução de incidência de fístulas e da morbidade pós-operatória no braço em que a droga foi utilizada. Estudos subsequentes, no entanto, não foram capazes de reproduzir esses resultados.

Na única metanálise disponível sobre o tema, Connor et al. ${ }^{8}$ analisando 22 estudos randomizados selecionaram 10 que preencheram os critérios de inclusão propostos, entre eles número mínimo de 50 pacientes incluídos por estudo. Desta forma total de 1918 pacientes foram examinados. Embora sem impacto nas taxas de mortalidade, o uso da somatostatina e de seus análogos foram eficazes em reduzir as taxas de morbidade geral e ainda àquelas relacionadas ao coto pancreático. A tentativa de se obter um subgrupo de pacientes nos quais os benefícios com o uso das drogas pudesse ser mais evidente não teve êxito em virtude da heterogenicidade entre os estudos. Os autores sugerem que em virtude dos dados atuais e ainda do custo da medicação ter se tornado menor, o uso rotineiro dessas drogas deva ser estimulado8. A maioria dos estudos orienta o seu uso pouco antes da operação na dose de $100 \mathrm{mcg}$ por via subcutânea de $8 / 8$ horas por sete dias de pós-operatório.

\section{Tratamento do coto pancreático}

Complicações decorrentes do remanescente pancreático após a remoção cirúrgica do AP como fístula, deiscência anastomótica, formação de abscesso, fístulas e hemorragia são a principal causa de morbimortalidade pós-operatória. Não é por outra razão que a anastomose pancreática é conhecida como o "calcanhar de Aquiles" da cirurgia pancreática. Mais que 80 tipos de maneira de se restabelecer a continuidade pancreatoentérica já foram descritas. Outras possibilidades como a oclusão do ducto por ligaduras, colas biológicas e ainda a pancreatectomia total foram tentadas no passado. No momento atual a anastomose do remanescente pancreático ao jejuno e ao estômago, são a prática corrente embora não haja definição com base na literatura sobre a melhor das duas técnicas ${ }^{18}$.

$\mathrm{Na}$ anastomose do remanescente pancreático com a alça jejunal existem três possibililidades: a) a anastomose "ducto-mucosa", na qual o ducto é anastomosado com a mucosa jejunal no primeiro plano de sutura e o parênquima pancreático à serosa da alça jejunal, no segundo plano de sutura; b) invaginação do coto pancreático na alça jejunal, tecnicamente mais fácil e prática e c) método de “telescopagem" na qual a alça jejunal é invertida e a invaginação do pâncreas permite que a cápsula pancreática se acole à serosa da alça jejunal. De todos os métodos apresentados a anastomose "ducto-mucosa" tem sido a mais extensamente utilizada após estudos experimentais e clínicos ${ }^{39}$.

É de importância assinalar que o uso de próteses na anastomose "ducto-mucosa", já foi tentado sem, no entanto, ter se demonstrado de valor na redução do risco de fístula pancreática ${ }^{42}$. Outra possibilidade técnica interessante e descrita por Machado et al. ${ }^{21}$ é a utilização de uma alça em "Y de Roux" separada da anastomose biliar de forma a impedir o refluxo de bile na presença de deiscência de anastomose pancreática ${ }^{21}$.

As vantagens em se utilizar o estômago para a anastomose com o coto pancreático são: excelente vascularização, facilidade do procedimento, acessibilidade endoscópica e via de drenagem separada da anastomose biliar. Embora tenham sido descrito índices menores de fístula pancreática com a pancreaticogastrostomia, em três estudos prospectivos disponíveis na literatura, não foi encontrado diferença na comparação com a pancreaticojejunostomia ${ }^{4,11,53}$.

$\mathrm{O}$ equivalente da anastomose pancreática após a ressecção dos tumores cefálicos é o fechamento do coto pancreático nas ressecções de tumores de localização no corpo e cauda pancreático. Não existem dados convincentes atualmente para se definir a melhor maneira de tratamento do coto pancreático. $\mathrm{O}$ fechamento por pontos, a utilização de grampeadores, a utilização de "patch" seroso e mesmo a anastomose pancreática, são possibilidades técnicas. Knaebel et al. ${ }^{19}$, a partir de 262 publicações, selecionaram 10 estudos para a revisão sistemática e seis para a metanálise na avaliação da melhor técnica de tratamento do coto pancreático pós-pancreatectomia corpo-caudal. A maioria das publicações utilizadas na revisão sistemática foi baseada em estudos observacionais com amostragem insuficiente, viés de seleção e definições não homogêneas de fístula pancreática, tornando conclusão inadequada sobre o assunto. A despeito disso, a metanálise apontou para uma tendência de menor incidência de fístula com o grampeamento ${ }^{19}$. Esse dado é discordante com estudo recente, prospectivo, não randomizado, com 302 pacientes consecutivos submetidos à pancreatectomia corpo-caudal no qual o fechamento do coto pancreático por meio de grampeador foi associado com significante maior índice de fístula pancreática ${ }^{17}$. São aguardados resultados de estudos prospectivos randomizados para elucidação do tema.

\section{Ressecção vascular?}

A cabeça e colo pancreático guardam íntima relação com o eixo mesentérico-portal sendo frequente a invasão dessa importante estrutura vascular pelo AP. Até 15 anos atrás a invasão deste eixo era considerada contraindicação formal para a ressecção pancreática, no entanto, com a publicação de grandes séries institucionais ao longo dos últimos anos incluindo pacientes com ressecções vasculares e com mesmos índices de morbidade que o procedimento 
padrão, a ressecção vascular tornou-se aceita. Muitos autores admitem hoje que esta invasão ocorre em virtude do local anatômico próximo ao de surgimento da lesão e não em virtude de maior agressividade do tumor. Desta forma a ressecção vascular associada torna-se necessária para que maior parcela dos pacientes se beneficie com os resultados da ressecção com margens negativas de tumor. Uma relação direta entre índice de ressecção vascular e margem negativa para tumor pode ser encontrada em séries cirúrgicas de pacientes com AP. Na maior casuística institucional com 1175 pacientes submetidos à duodenopancreatectomia por AP no Johns Hopkins Medical Institutions, a ressecção vascular foi realizada em apenas $4 \%$ dos pacientes e a taxa de margem comprometida por tumor foi de $42 \%{ }^{48}$. Esses dados contrastam com outras séries nas quais a ressecção vascular é realizada em maior proporção de pacientes. Raut et al. ${ }^{32}$ e Yekebas et al ${ }^{54}$ encontraram taxa de margem comprometida respectivamente em $16,7 \%$ e $14,2 \%$ dos seus pacientes, provavelmente em virtude de maior índice de ressecção vascular $(36,1 \%$ e $23,2 \%)$. Ainda segundo os autores, uma vez com margem cirúrgica negativa para tumor os índices de sobrevida foram semelhantes com ou sem a ressecção vascular.

Em revisão sistemática, única na literatura, que analisou 52 publicações de pacientes submetidos à pancreatectomia com ressecção do eixo, os dados de sobrevida não foram tão animadores. Foram identificados 1646 pacientes dos quais, $88,6 \%$ submeteram-se à ressecção venosa com anastomose primária enquanto $9,7 \%$ foram reconstruídos com enxerto autólogo de veia. A sobrevida em 1, 3 e 5 anos foi respectivamente de 50,16 e $7 \%$. Na análise mais detalhada desse grupo de pacientes pode-se observar alta taxa de metástase linfonodal $(67,4 \%)$ e de margens de ressecção livres de tumor $(39,8 \%)$. Isso comprova que a ressecção venosa deve ser indicada apenas nos casos em que a adição desse procedimento viabilize a ressecção com margens livres de tumor do contrário, o procedimento não será benéfico aos pacientes ${ }^{40}$.

Quanto à ressecção arterial (artéria mesentérica superior e hepática) nos pacientes com AP os dados atuais sugerem maior morbi-mortalidade com sobrevida inferior quando esse procedimento é comparado à pancreatectomia padrão. Em estudo que incluiu 201 pacientes submetidos à ressecção vascular associado à pancreatectomia, Nakao et al. ${ }^{27}$ observaram taxa de mortalidade de $35,7 \%$ dos 14 pacientes submetidos à ressecção arterial associada a ressecção de veia porta comparada a 2,7\% dos 186 pacien- tes submetidos apenas à ressecção venosa27. Mortalidade alta (17\%) também foi encontrado por Stitzenberg et al.44 em 12 pacientes submetidos à ressecção arterial durante procedimento de pancreatectomia por AP não sendo encontrado nenhum caso de sobrevida superior a cinco anos. Desta forma, a invasão das artérias mesentérica ou hepática, é considerada no momento atual, contraindicação para a ressecçãa ${ }^{44}$.

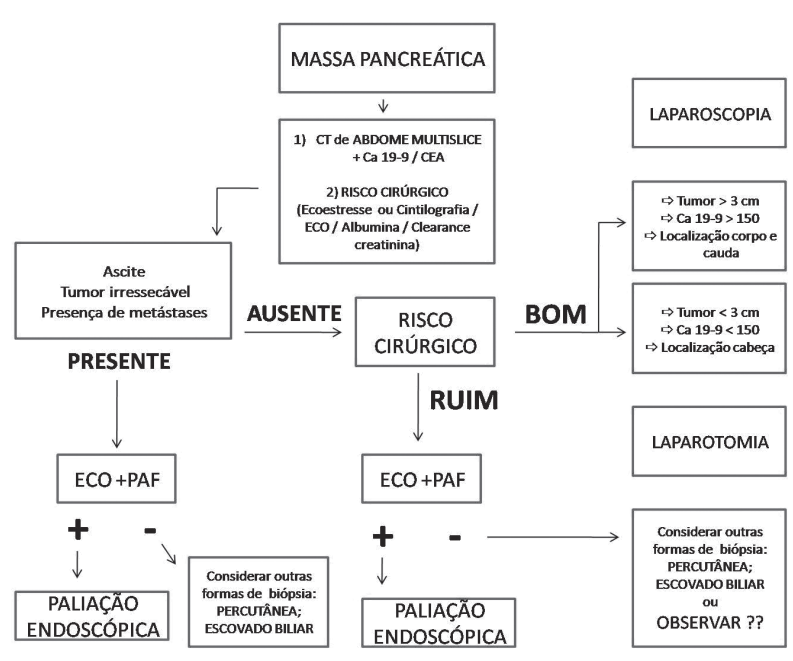

FIGURA 1 - Algoritmo para abordagem do AP

\section{CONCLUSÕES}

A abordagem diagnóstica de um paciente com diagnóstico de massa em topografia de pâncreas constitui ainda hoje complexo problema clínico. Isso ocorre devido à dificuldade na obtenção de amostra de tecido para o diagnóstico associado aos riscos da ressecção cirúrgica em pacientes que comumente estão na sexta década de vida. Hoje em dia na suspeita de adenocarcinoma de pâncreas é possível realizar estadiamento muito próximo do real sem a necessidade da exploração cirúrgica sistemática em virtude da disponibilidade na prática de exames modernos e eficientes. Isso permite que paliação menos invasiva seja praticada na maioria dos pacientes com lesões avançadas e incuráveis. Nos em que a cura é possível, a operação deve ser realizada objetivando-se, essencialmente, a remoção da lesão com margens livres e com aceitáveis índices de morbi-mortalidade. 
Amico EC, Barreto JSS, Dantas-Filho AM, Araújo-Filho I. Diagnosis, staging and treatment of pancreatic adenocarcinoma. ABCD Arq Bras Cir Dig 2008;21(4): 192-200

ABSTRACT - Introduction-Pancreatic adenocarcinoma is one of the solid tumors with bad prognosis, and surgical treatment is the only one potentially curative. Methods - In majority of patients the condition is diagnosed when already it is in advanced phase, commonly with metastatic disease. The introduction of new diagnostic methods associated with improvement of the already existed, created controversy in the relationship to the diagnosis and tumor staging. Conclusion - Thus, the role of surgery in palliative treatment and the technical aspects of the removal of the localized lesions, are far from having consensus in practice. A survey of the literature on the controversial aspects and an algorithm for the approach in patients with suspected pancreatic tumor are presented.

HEADINGS - Pancreas. Adenocarcinoma. Diagnosis. Surgery.

\section{REFERÊNCIAS}

1. Alexakis N, Ghaneh P, Neoptolemos JP. In: Hans Beger, 2 ed. The pancreas: an integrated textbook of basic sciences, medicine, and surgery, 2008. 573-82.

2. Alexakis N, Halloran C, Raraty M, Ghaneh P, Sutton R, Neoptolemos JP. Current standards of surgery for pancreatic câncer. Br J Surg. 2004; 91: 1410-27.

3. Artifon EL, Sakai P, Cunha JE, Dupont A, Filho FM, Hondo FY, Ishioka S, Raju GS. Surgery or endoscopy for palliation of biliary obstruction due to metastatic pancreatic câncer. Am J Gastroenterol. 2006 Sep;101(9):2031-7.

4. Bassi C, Falconi M, Molinari E, Salvia R, Butturini G, Sartori N, Mantovani W, Pederzoli P Reconstruction by pancreati- cojejunostomy versus pancreaticogastrostomy following pancreatectomy: results of a comparative study. Ann Surg 2005; 242:767-71.

5. Cameron JL, Riall TS, Coleman J, Belcher KA. One consecutive pancreaticoduodenectomies. Ann Surg. 2006; 244:10-5.

6. Clarke DL, Thomson SR, Madiba TE, Sanyika C. Preoperative imaging of pancreatic cancer: a management-oriented approach. J Am Coll Surg. 2003 Jan;196(1):119-29.

7. Conlon $\mathrm{KC}$, Brennan MF. Laparoscopy for staging abdominal malignancies. Adv Surg. 2000;34:331-50.

8. Connor S, Alexakis N, Garden OJ, Leandros E, Bramis J, Wigmore SJ. Metaanalysis of the value of somatostatin and its analogues in reducing complications associated with pancreatic surgery. Br J Surg. 2005 Sep;92(9):1059-67.

9. DeWitt J, Devereaux B, Chriswell M, McGreevy K, Howard T, Imperiale TF, Ciaccia D, Lane KA, Maglinte D, Kopecky K, LeBlanc J, McHenry L, Madura J, Aisen A, Cramer H, Cummings O, Sherman S. Comparison of endoscopic ultrasonography and multidetector computed tomography for detecting and staging pancreatic câncer. Ann Intern Med. 2004 Nov 16;141(10):753-63.

10. Di Stasi M, Lencioni R, Solmi L, Magnolfi F, Caturelli E, De Sio I, Salmi A, Buscarini L. Am J Gastroenterology. 1998; 8: 1329-1333.

11. Duffas JP, Suc B, Msika S, Fourtanier G, Muscari F, Hay JM, Fingerhut A, Millat B, Radovanowic A, Fagniez PL A controlled randomized multicenter trial of pancreatogastrostomy or pancreatojejunostomy after pancreatoduodenectomy. Am J Surg; 2005; 189:720-29.

12. Espat NJ, Brennan MF, Conlon KC. Patients with laparoscopically staged unresectable pancreatic adenocarcinoma do not require subsequent surgical biliary or gastric bypass. J Am Coll Surg. 1999;188:649-55.

13. Ferrone CR, Haas B, Tang L, Coit DG, Fong Y, Brennan MF, Allen PJ. The influence of positive peritoneal cytology on survival in patients with pancreatic adenocarcinoma. J Gastrointest Surg. 2006 Dec;10(10):1347-53.

14. Heinrich S, Goerres GW, Schäfer M, Sagmeister M, Bauerfeind P, Pestalozzi BC, Hany TF, von Schulthess GK, Clavien PA. Positron emission tomography/ computed tomography influences on the management of resectable pancreatic cancer and its cost-effectiveness. Ann Surg. 2005 Aug;242(2):235-43.

15. INCA. Instituto Nacional de Câncer. http://www.inca.gov.br/conteudo_view. asp?id=337.

16. Kaassis M, Boyer J, Dumas R, Ponchon T, Coumaros D, Delcenserie R, Canard JM, Fritsch J, Rey JF, Burtin P. Plastic or metal stents for malignant stricture of the common bile duct? Results of a randomized prospective study. Gastrointest Endosc. 2003; 57:178-82.

17. Kleeff J, Diener MK, Z'graggen K, Hinz U, Wagner M, Bachmann J, Zehetner J, Muller MW, Friess H, Buchler MW. Distal Pancreatectomy Risk Factors for Surgical Failure in 302 Consecutive Cases. Annals of Surgery 2007; 245:573-82.

18. Kleespies A, Albertsmeier M, Obeidat F, Seeliger H, Jauch KW, Bruns CJ. The challenge of pancreatic anastomosis. Langenbecks Arch Surg 2008; 393:459-71.

19. Knaebel HP, Diener MK, Wente MN, Buchler MW, Seiler CM. Systematic review and meta-analysis of technique for closure of the pancreatic remnant after distal pancreatectomy. British Journal of Surgery 2005; 92: 539-46.

20. Lillemoe KD, Cameron JL, Hardacre JM, Sohn TA, Sauter PK, Coleman J, Pitt HA, Yeo CJ. Is prophylactic gastrojejunostomy indicated for unresectable periampullary cancer? A prospective randomized Trial. Ann Surg. 1999 Sep;230(3):322-8.

21. Machado MC, da Cunha JE, Bacchella T, Bove P. A modified technique for the reconstruction of the alimentary tract after pancreatoduodenectomy. Surg Gynecol Obstet. 1976 Aug;143(2):271-2.
22. Maluf-Filho, F; Dotti, CM; Farias, AQ; Kupski, C; Chaves, DM; Artifon, E; Nakao, F; Rossini, GF; Paulo, GA; Ardengh, JC; Silva, JEF; Rossini, L; Lima, LFP; Averbach, M; Cury, MS; D'Aassunção, MA; Silva, MC; Ney, MV; Spinosa, S; Matuguma, SE; Guaraldi, S; Arantes, V; Mello, VH. I Consenso Brasileiro de Ecoendoscopia. Arq Gastroenterol. 2007; 44:353-358.

23. Merchant NB, Conlon KC. Laparoscopic evaluation in pancreatic cancer. Semin Surg Oncol. 1998 Oct-Nov;15(3):155-65.

24. Meszoely IM, Lee JS, Watson JC, Meyers M, Wang H, Hoffman JP. Peritoneal cytology in patients with potentially resectable adenocarcinoma of the pâncreas. Am Surg. 2004 Mar;70(3):208-13.

25. Michalski CW, Kleeff J, Wente MN, Diener MK, Büchler MW, Friess H. Systematic review and meta-analysis of standard and extended lymphadenectomy in pancreaticoduodenectomy for pancreatic cancer. Br J Surg. 2007 Mar;94(3):265-73.

26. Morganti AG, Brizi MG, Macchia G, Sallustio G, Costamagna G, Alfieri S, Mattiucci GC, Valentini V, Natale L, Deodato F, Mutignani M, Doglietto GB, Cellini N. The prognostic effect of clinical staging in pancreatic adenocarcinoma. Ann Surg Oncol. 2005 Feb;12(2):145-51.

27. Nakao A, Takeda S, Inoue S, Nomoto S, Kanazumi N, Sugimoto H, Fujii T. Indications and techniques of extended resection for pancreatic câncer. World J Surg. 2006 Jun;30(6):976-82.

28. Nimura Y, Nagino M, Kato H, Miyagawa S, Yamaguchi A, Kinoshita T et al. Regional versus extended lymph node dissection in radical pancreaticoduodenectomy for pancreatic cancer: a multicenter, randomized controlled trial. HPB 2004; 6(Suppl 1): 2 (Abstract).

29. Pancreatic Section of the British Society of Gastroenterology. Guidelines for the management of patients with pancreatic cancer periampullary and ampullary carcinomas. Gut. 2005; 54(suppl):1-16.

30. Parsons CM, Sutcliffe JL, Bold RJ. Preoperative evaluation of pancreatic adenocarcinoma. J Hepatobiliary Pancreat Surg. 2008;15(4):429-35.

31. Pedrazzoli S, DiCarlo V, Dionigi R, Mosca F, Pederzoli P, Pasquali C et al. Standard versus extended lymphadenectomy associated with pancreatoduodenectomy in the surgical treatment of adenocarcinoma of the head of the pancreas: a multicenter, prospective, randomized study. Lymphadenectomy Study Group. Ann Surg 1998; 228: 508-517.

32. Raut CP, Tseng JF, Sun CC, Wang H, Wolff RA, Crane CH, Hwang R, Vauthey JN, Abdalla EK, Lee JE, Pisters PW, Evans DB. Impact of resection status on pattern of failure and survival after pancreaticoduodenectomy for pancreatic adenocarcinoma. Ann Surg. 2007 Jul;246(1):52-60.

33. Rocha-Lima CM. New directions in the management of advanced pancreatic câncer: a review. Anti-Cancer Drugs 2008; 19: 435-446.

34. Rodriguez JR, Warshaw AL, Del Castillo CF. In: Hans Beger, 2 ed. The pancreas: an integrated textbook of basic sciences, medicine, and surgery, 2008. 643-47.

35. Royal RE, Wolff RA, Crane CH. Pancreatic Cancer. In: DeVita, Hellman, and Rosenberg's Cancer: Principles \& Practice of Oncology, Eighth Edition. Lippincott Williams \& Wilkins, 2008. p. 1086-1124.

36. Schembre. In: Hans Beger, 2 ed. The pancreas: an integrated textbook of basic sciences, medicine, and surgery, 2008. 621-28.

37. Schlieman MG, Ho HS, Bold RJ. Utility of tumor markers in determining resectability of pancreatic cancer. Arch Surg. 2003 Sep;138(9):951-5.

38. Sewnath ME, Karsten TM, Prins MH, Rauws EJ, Obertop H, Gouma DJ. A meta-analysis on the efficacy of preoperative biliary drainage for tumors causing obstructive jaundice. Ann Surg. 2002 Jul;236(1):17-27.

39. Shrikhande SV, Qureshi SS, Rajneesh N, Shukla PJ. Pancreatic Anastomoses after Pancreaticoduodenectomy: Do We Need Further Studies? World J Surg 2005; 29: 1642-9.

40. Siriwardana HP, Siriwardena AK. Systematic review of outcome of synchronous portal-superior mesenteric vein resection during pancreatectomy for cancer. $\mathrm{Br}$ J Surg. 2006 Jun;93(6):662-73.

41. Smith CD, Behrns KE, van Heerden JA, Sarr MG. Radical pancreatoduodenectomy for misdiagnosed pancreatic mass. Br J Surg. 1994;81:585-9.

42. Soriano A, Castells A, Ayuso C, Ayuso JR, de Caralt MT, Ginès MA, Real 
MI, Gilabert R, Quintó L, Trilla A, Feu F, Montanyà X, Fernández-Cruz L, Navarro S. Preoperative staging and tumor resectability assessment of pancreatic cancer: prospective study comparing endoscopic ultrasonography, helical computed tomography, magnetic resonance imaging, and angiography. Am J Gastroenterol. 2004 Mar;99(3):492-501.

43. Stefanidis D, Grove KD, Schwesinger WH, Thomas CR Jr. The current role of staging laparoscopy for adenocarcinoma of the pancreas: a review. Ann Oncol. 2006 Feb;17(2):189-99.

44. Stitzenberg KB, Watson JC, Roberts A, Kagan SA, Cohen SJ, Konski AA, Hoffman JP. Survival after pancreatectomy with major arterial resection and reconstruction. Ann Surg Oncol. 2008 May;15(5):1399-406.

45. Tran KTC, Smeenk HG, Van Eijck CHJ, Kazemier G, Hop WC, Greve JWG, Terpstra OT, Zijlstra JA, Klinkert P, Jeekel H. Pylorus preserving pancreaticoduodenectomy versus standard whipple procedure a prospective, randomized, multicenter analysis of 170 patients with pancreatic and periampullary tumors. Ann Surg 2004;240: 738-745.

46. Van der Gaag NA, de Castro SM, Rauws EA, Bruno MJ, van Eijck CH, Kuipers EJ, Gerritsen JJ, Rutten JP, Greve JW, Hesselink EJ, Klinkenbij1 JH, Rinkes IH, Boerma D, Bonsing BA, van Laarhoven CJ, Kubben FJ, van der Harst E, Sosef MN, Bosscha K, de Hingh IH, Th de Wit L, van Delden OM, Busch OR, van Gulik TM, Bossuyt PM, Gouma DJ. Preoperative biliary drainage for periampullary tumors causing obstructive jaundice; Drainage vs. (direct) Operation (DROP-trial). BMC Surg. 2007 Mar 12;7:3.

47. Van Heek NT, De Castro SM, van Eijck CH, van Geenen RC, Hesselink EJ, Breslau PJ, Tran TC, Kazemier G, Visser MR, Busch OR, Obertop H, Gouma DJ. The need for a prophylactic gastrojejunostomy for unresectable periampullary cancer: a prospective randomized multicenter trial with special focus on assessment of quality of life. Ann Surg. 2003 Dec;238(6):894-902.

48. Winter JM, Cameron JL, Campbell KA, Arnold MA, Chang DC, Coleman J, Hodgin MB, Sauter PK, Hruban RH, Riall TS, Schulick RD, Choti MA, Lillemoe KD, Yeo CJ. 1423 pancreaticoduodenectomies for pancreatic cancer: A single-institution experience. J Gastrointest Surg. 2006 Nov;10(9):1199-210.
49. Winter JM, Cameron JL, Campbell KA, Chang DC, Riall TS, Schulick RD, Choti MA, Coleman J, Hodgin MB, Sauter PK, Sonnenday CJ, Wolfgang CL, Marohn MR, Yeo CJ. Does pancreatic duct stenting decrease the rate of pancreatic fistula following pancreaticoduodenectomy? Results of a prospective randomized trial. J Gastrointest Surg. 2006 Nov;10(9):1280-90.

50. Wolfson D, Barkin JS, Chari ST, Clain JE, Bell RH, Alexakis N, Neoptolemos JP. Management of Pancreatic Masses. Pancreas 2005; 31: 203-217.

51. Yachida S, Fukushima N, Sakamoto M, Matsuno Y, Kosuge T, Hirohashi S. Implications of peritoneal washing cytology in patients with potentially resectable pancreatic cancer. Br J Surg. 2002 May;89(5):573-8.

52. Yeo CJ, Cameron JL, Lillemoe KD, Sohn TA, Campbell KA, Sauter PK et al. Pancreaticoduodenectomy with or without distal gastrectomy and extended retroperitoneal lymphadenectomy for periampullary adenocarcinoma, part 2: randomized controlled Trial evaluating survival, morbidity, and mortality. Ann Surg 2002; 236: 355-366; discussion 366-368.

53. Yeo CJ, Cameron JL, Maher MM, Sauter PK, Zahurak ML, Talamini MA, Lillemoe KD, Pitt HAA prospective randomized trial of pancreaticogastrostomy versus pancreatico- jejunostomy after pancreaticoduodenectomy. Ann Surg 1995; 222:580- 588 discussion 588-92.

54. Yekebas EF, Bogoevski D, Cataldegirmen G, Kunze C, Marx A, Vashist YK, Schurr PG, Liebl L, Thieltges S, Gawad KA, Schneider C, Izbicki JR. En bloc vascular resection for locally advanced pancreatic malignancies infiltrating major blood vessels: perioperative outcome and long-term survival in 136 patients. Ann Surg. 2008 Feb;247(2):300-9.

Fonte de financiamento: não há Conflito de interesse: não há Recebido para publicação: 10/06/2008 Aceito para publicação: 25/09/2008 